\title{
Uraemia disarms neutrophils against Candida
}

ce

Our work provides a rationale for future clinical studies to test the use of GSK3 $\beta$ inhibitors to treat or prevent systemic candidiasis in patients with kidney disease. difficult to control in these patients."

The researchers modelled kidney damage-induced uraemia by injecting mice with a nephrotoxic derivative of aristolochic acid (AAI); mice injected with AAII, which is also mutagenic but does not cause kidney damage, or with phosphate-buffered saline were used as controls. Only the mice injected with AAI (AAI-mice) developed uraemia after 3 days (blood urea nitrogen levels peaked after 6-10 days). When challenged with an intravenous C. albicans injection 4 days after the induction of kidney disease, AAI-mice were

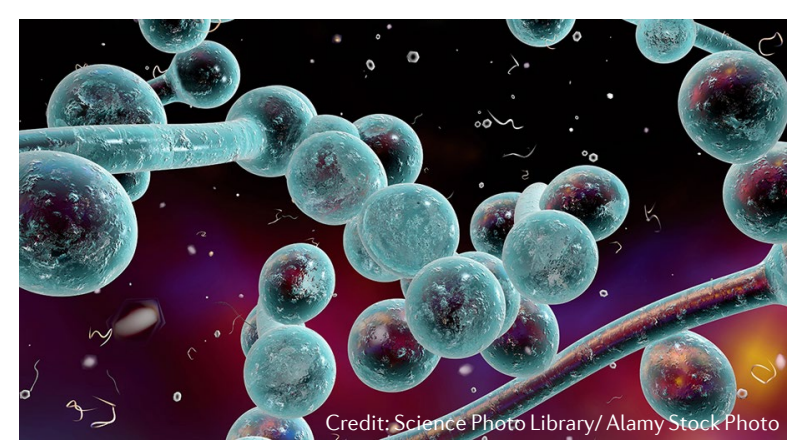

more susceptible to infection and had higher mortality than controls. By contrast, in the unilateral ureteral obstruction mouse model, in which kidney damage did not lead to uraemia, fungal load did not differ between mice with kidney dysfunction and their controls.

Neutrophils have a key role in antifungal immunity, and the researchers therefore investigated whether uraemia affected the ability of these innate immune cells to kill C. albicans. In vitro, exposure to serum from AAI-mice (AAI-serum), but not to similar concentrations of AAI alone, reduced neutrophil cytoplasmic reactive oxygen species (ROS) production and impaired C. albicans killing. Accordingly, in infected AAI-mice, the frequency of kidney-infiltrating neutrophils that had internalized and killed C. albicans was reduced compared with controls. ROS production in neutrophils requires oxygen and $\mathrm{NADPH}$, which is produced via two glucose-dependent processes glycolysis and the pentose phosphate pathway. Compared with AAI alone, exposure to AAI-serum reduced C. albicans-induced neutrophil glucose uptake and glycolysis; levels of the GLUT1 glucose transporter were also reduced. In vivo, glucose uptake in neutrophils from AAI-mice was also reduced, and healthy mice infected with C. albicans that were treated with a GLUT1 inhibitor had a higher fungal burden and lower neutrophil ROS levels than untreated mice.

The researchers noted that exposure to AAI-serum in vitro inhibited AKT activation, reduced phosphorylation-induced inhibition of GSK $3 \beta$ and inhibited mTORC1 activity in neutrophils. These observations suggested that, by disrupting AKT activation, AAI-serum promoted GS3K $\beta$ activation, which could reduce GLUT1 expression by inhibiting mTOR. The researchers then tested the effects of GSK3 $\beta$ inhibitors, including clinically approved lithium chloride ( $\mathrm{LiCl})$, on neutrophils. Blocking GSK3 $\beta$ in vitro restored GLUT1 expression, glucose uptake and C. albicans killing in neutrophils cultured with AAI-serum. Similarly, infected AAI-mice treated with $\mathrm{LiCl}$ had higher neutrophil glucose uptake, increased ROS production, lower fungal burden and improved survival compared with untreated controls. "The use of GSK3 $\beta$ inhibitors to restore fungicidal activity of neutrophils in a pre-clinical model of kidney disease is a novel approach," notes Biswas. "Our work provides a rationale for future clinical studies to test the use of GSK $3 \beta$ inhibitors to treat or prevent systemic candidiasis in patients with kidney disease."

Human neutrophils from healthy volunteers that were cultured with C. albicans in the presence of pre-dialysis serum from patients with kidney failure produced less ROS and were less fungicidal than neutrophils cultured in the presence of post-dialysis serum from the same patients. In vitro treatment with $\mathrm{LiCl}$ minimized the neutrophil defects induced by the pre-dialysis serum.

"We plan to identify which uraemic toxins cause neutrophil dysfunction in patients with kidney disease, especially in patients treated with dialysis," explains Biswas. "We also want to define how uraemia or uraemic toxins suppress adaptive immunity to inform vaccine development strategies for these patients."

Monica Wang

ORIGINAL ARTICLE Jawale, C. V. et al. Restoring glucose uptake rescues neutrophil dysfunction and protects against systemic fungal infection in mouse models of kidney disease. Sci. Transl. Med. 12, eaay5691 (2020) 